\title{
FiAAR: An Augmented Reality Firetruck Equipment Assembly and Configuration Assistant Technology
}

\author{
Fahmi Bellalouna $^{1}$, Mika Luimula ${ }^{2}$, Panagiotis Markopoulos ${ }^{3}$, Evangelos Markopoulos ${ }^{4,1}$, Franco Zipperling ${ }^{5}$ \\ ${ }^{1}$ Karlsruhe University of Applied Sciences, Karlsruhe Germany \\ ${ }^{1}$ Turku University of Applied Sciences, Turku Finland \\ ${ }^{3}$ University of the Arts London, London UK \\ ${ }^{4}$ University College London, London, UK. \\ ${ }^{5}$ Rosenbauer GmbH, Karlsruhe, Germany \\ Fahmi.bellalouna@hs-karlsruhe.de; mika.luimula@turkuamk.fi; p.markopoulos1@arts.ac.uk; \\ e.markopoulos@ucl.ac.uk; Franco.Zipperling@rosenbauer.com
}

\begin{abstract}
Augmented reality (AR) is the technology that expands the physical world by enhancing the objects that reside in the real world with computer generated perceptual information to provide interactive experience in a real-world environment. AR is used effectively in many business sectors such as the engineering, education and training, medicine, logistics and transport, and others. Rescue services is one of the challenging areas where the use of AR technology has extremely high demands for robustness and ease of use. In this paper, we have introduced two augmented reality versions for the FiAAR project developed by Karlsruhe University of Applied Sciences. The first version is developed for Realmax HMT-1 device with hands free interaction utilizing speech recognition. The second version in turn focuses on hand recognition and it's developed for Realwear Qian device with Leap Motion sensor. Our intention is to show the potential of current AR technologies in demanding use cases.
\end{abstract}

Keywords: Augmented Reality, hand tracking, speech recognition, firetruck, training, safety.

\section{INTRODUCTION}

Augmented reality (AR), is the technology which allows computer generated virtual images to overlay physical objects in real time. The difference between AR and VR (Virtual Reality) is that AR allows the user to interact with the virtual images using real objects in a seamless way, while in VR the user is completely immersed in a virtual environment. [1] AR is used effectively in many business sectors such as the engineering, medicine, logistics and transport, and others but two sectors are mostly related with the technology presented in this paper. The first one is the automotive sector and the second is the education sector.

The automotive sector is one of the major and early believers in AR that continues to support the development of the technology [2]. Many automobile manufacturers integrated AR with modest heads-up displays (HUDs) that project, in the corner of the windshield, information such as speed and mileage, while others invested in more ambitious AR projects aiming to turn the entire windshield into a hologram that can display GPS directions, road conditions, weather alerts and real-time information about the automobile surroundings [3].

Similar to the automotive sector, the AR market size, awareness and adaptation in the training and education sector indicates impressive growth as well [4]. AR offers significant potential and benefits for the augmentation of teaching and learning environments [5]. These can be related with the student's engagement, simulation and motivation [6], the ability to teach subjects where students could not feasibly gain real-world first-hand experience [7], to increase student-instructor collaboration [8], to help students take control of their learning pace and path and to create authentic learning environments suitable to the learning style of each student or learning objective.

The interactive nature of the technology contributes to what the classroom of the future can be. Furthermore, the development of AR interactive games and activities can reinforce key concepts and increase students learning effectiveness. Finally, and most importantly, AR can help students with disabilities overcome challenges in the classroom [9].

Driven by the training and educational applications of $\mathrm{AR}$ and its innovative applications and adaptation on the automobiles industry we developed the FiAAR technology composed from two versions of an AR application (with speech and hand recognition) in the sector of fire safety. This research was leaded by the Karlsruhe University of Applied and the Turku University of Applied Sciences that contributed on the technical implementation of technology. Furthermore, Rosenbauer contributed with the technical expertise and application's requirements elicitation, verification and system testing.

\section{THE AUGMENTED REALITY INDUSTRY}

Our efforts to create an academia-industry research joint research initiative and develop in parallel the two versions of the FiAAR technology for such a niche industry and market, was also inspired by the fast and impressive growth of AR globally in terms of markets share, applications users and developers. The AR market forecast is expected to reach $\$ 70-\$ 75$ billion in revenue by 2023. Only the sales of mobile AR devices and smart glasses are to reach above the 2.5 billion units by 2023 , creating tremendous opportunities for innovative $A R$ applications [10]. 
The healthcare industry is expected to lead the AR growth as it provides the most opportunities to patients and health providers. Medical students use AR training to practice surgical techniques while physicians are able to observe the patient's vitals and refer to MRI or CT scanning images [11].

The defense industry has also demonstrated impressive, extensive and expensive use of the AR technology. The F35 fighter jet's helmet costs $400.000 \$$ and allows the pilot to look through the plane itself. It also identifies and provides information about any object in the pilot's field of view [12].

However, what made AR popular to the world was the Pokémon GO game which has over 1 billion downloads (14\% of the world's population) [13]. The game reached $\$ 207$ million in revenue on its first month and reaching a peak of 45 million daily users worldwide. The game remains highly profitable over the years with $\$ 200,000$ daily revenue as of April 2019.

Today, $70-75 \%$ of people aged $16-44$ are aware of AR, and about $90 \%$ are aware of VR [14]. The popularity of AR extends also to the Mid-Sized Companies with $88 \%$ of them to using AR today in some capacity [15] mostly on advertising efforts to boost customer engagement.

This generated the need for more AR applications and AR development technologies. In 2017, Apple's ARKit made it possible for mobile app developers to design and develop innovative AR experiences for iOS devices. A year later, Google introduced ARCode and helped AR app developers to address the Android device market as well [16]. These and other technologies resulted in the creation of thousands of AR Mobile applications. Microsoft followed this trend and invested massively in both AR and VR, gaining over 10,000 related patents. Microsoft's firstgeneration AR glasses, HoloLens, already sold over 50,000 units [17] fundamentally transformed the service industry, among others. Field service technicians face significant challenges when working with thousand equipment varying in provider's specifications. Integrating AR with sophisticated sensors delivers realtime information on equipment status helping to identify parts that need replacement or highlighting potential risks not visible with naked eyes [18].

This tremendous growth of AR created a new entrepreneurial revolution inspiring many startups to direct their expertise to the AR market. Today more than 3.000 AR focused startups are listed on the Angel list [19]. Many investment analysts lean towards publicly traded companies which induce substantial growth due to $A R$ innovation.

\section{FIRETRUCK EQUIPMENT ASSEMBLY AND CONFIGURATION CHALLENGES.}

The FiAAR AR application presented in this paper derived from a real industry need. Until now, planning the interior of a locker room of a fire truck was only possible after the turntable ladder had been manufactured. The customer was asked to come to the factory in order to work with the interior fitting specialists to determine the position and location of the firefighters equipment.
However various problems have risen from this procedure. In particular, the timely procurement of rotating bearings and extension compartments for the firefighters equipment has repeatedly proven to be a problem and has often led to late delivery of the turntable ladder to the customer.

One solution was seen in completely detaching the planning process of the interior of the locker room from the completion of the turntable ladder. As soon as the Locker room has been designed, these 3D data could be used for virtual planning of the interior. This would allow to start the planning process with the customer at a very early stage.

This method also allows to order all swivel bearings and extension compartments at a very early stage. This ability has a huge positive effect on the manufacturing side.

Now, the required swivel bearings and extensions compartments can be mounted within the assembly line which helps to reduce the assembly costs significantly.

The virtual planning is to be carried out using AR technology. AR has a decisive advantage over VR: The interior fittings planned in AR can be projected into the equipment room during the interior fittings. This gives the fitter an exact specification of the position at which firefighting equipment should be installed. Furthermore, during the check of the interior by the customer, the AR planning can be projected over the real interior. This allows a very good comparison of the implementation of the planned interior fittings with the real interior fittings.

\section{THE FIAAR AUGMENTED REALITY TECHNOLOGY.}

The firefighting equipment (e.g. power saw, axe, fire truck, hoses) is housed in various equipment boxes in a firetruck. There is a wide variety of equipment configurations that can be stored in the different equipment cases with different positions (Fig. 1).

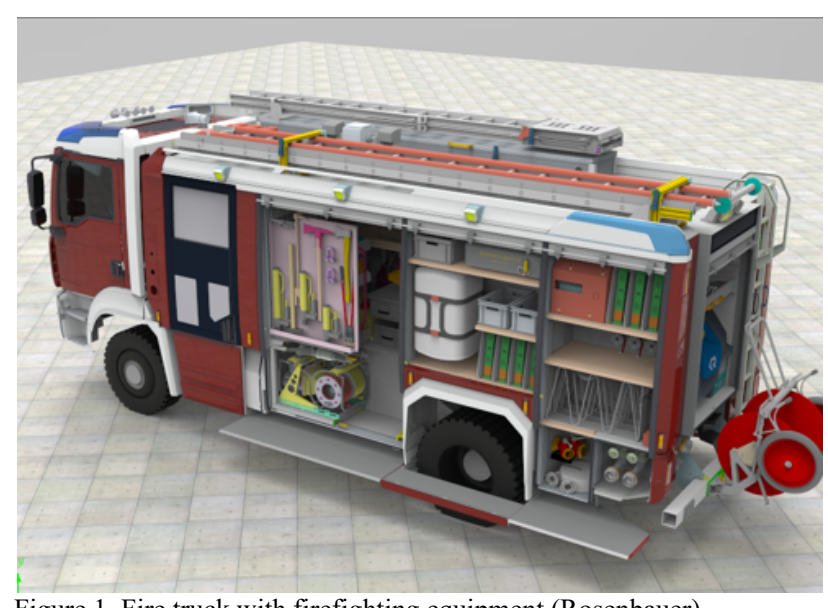

Figure 1. Fire truck with firefighting equipment (Rosenbauer)

The equipment configuration is determined in collaboration with the fire department during the sales process individually. Currently, the assembly process of the firefighting equipment is based on paper 2D drawings, which depict the equipment and its location in the firetruck. 
However, this process is very elaborate and error-prone due to the high variety of the equipment, its storage and positioning.

This process is particularly difficult for inexperienced employees and leads to high error rates while the assembly process. This causes high numbers of customer complaints, which lead to high costs for guarantee and after sales service, as well as customer dissatisfaction.

In order to improve the quality and to reduce the error rate during the assembly process, the FiAAR, an AR-based Firetruck Equipment Assembly and Configuration Assistant was developed in cooperation with the firefighter equipment and truck manufacturer Rosenbauer Karlsruhe $\mathrm{GmbH} \& \mathrm{Co}$. KG.

The AR application is intended to display the firefighting equipment virtually in the empty physical equipment cases using a mobile device (e.g. tablet, smartphone). This should make it easier for new employees to get started with assembly process and support them along the assembly process of a fire truck. In addition, the AR application can be used to visualize the final assembly state of the firefighting equipment to the fire department in the earlier sales process phase. This has the advantage of avoiding misunderstandings during the requirements management process and identifying change requests at an early product planning stage.

\section{FUNCTIONS OF THE FIAAR TECHNOLOGY}

In order to identify the needed functions for the AR application, several workshops were organized with the employees at the assembly shop floor of the company Rosenbauer. During these workshops the typical everyday experiences of the employees were analyzed and documented as UX (user experiences). The UX describes the design of the UI (user interface) and the user interaction with the AR application, which they were implemented as AR functions.

To start the AR-based assembly process, the QR code of the fire truck case that have to be assembled has to be scanned (Fig. 2).

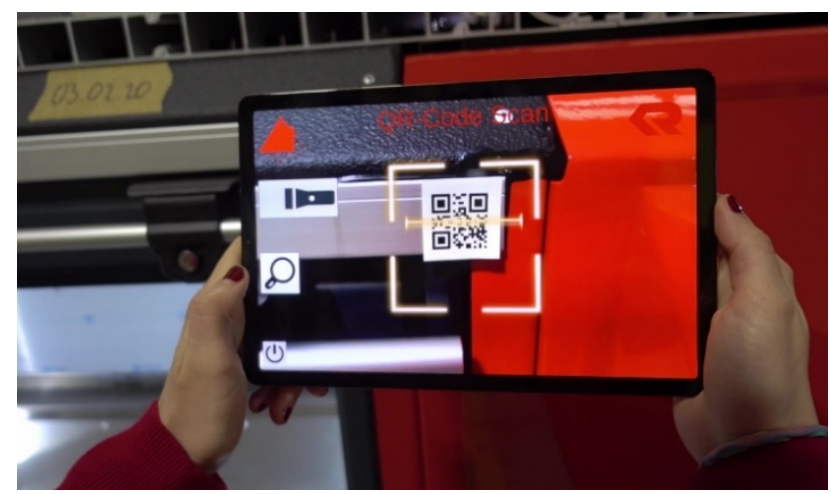

Figure 2. QR code scanning

Based on this information the virtual data of the firefighting equipment will be loaded while the following steps. Afterwards, an AR visual target for the concerned equipment box will appear, which can serve to identify and track the real box in the fire truck (Fig. 3).

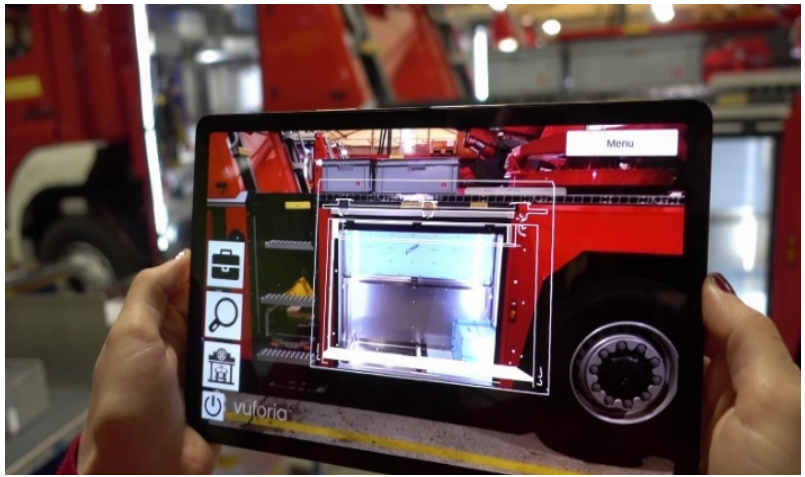

Figure 3. AR target to recognize the real firefighting equipment box

After the identification of the real equipment box, the virtual graphic data of the box (Fig. 4) and the included firefighting equipment will be displayed in the $3 \mathrm{D}$ space in the right position (Fig. 5).

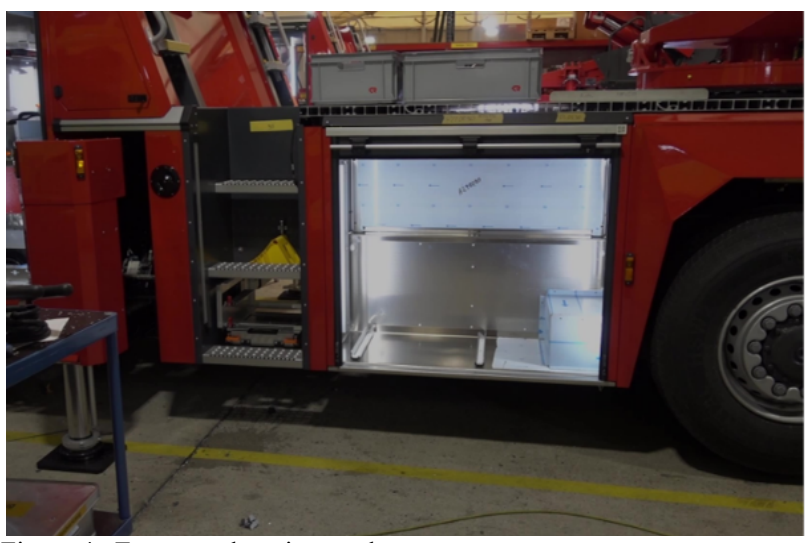

Figure 4. Empty real equipment box

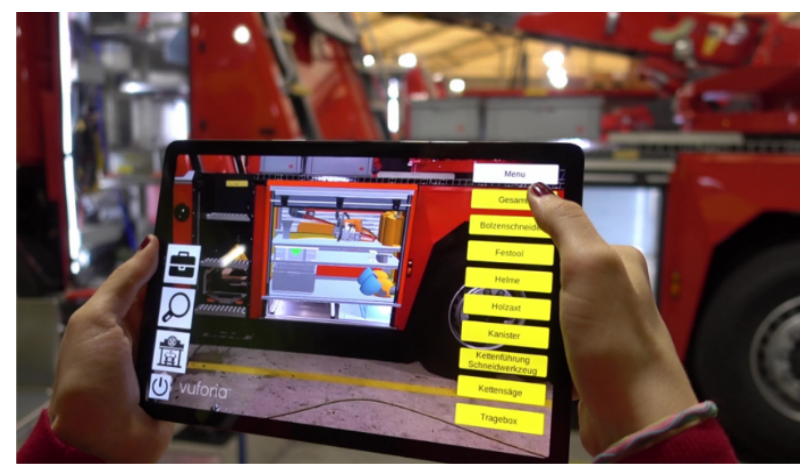

Figure 5. Visualization of the virtual graphic data of the box and the included firefighting equipment

Using the camera of the AR device, an uninterrupted model tracking can be ensured even though the user move around the equipment box.

Furthermore, the firefighting equipment will be shown as tree structure that can be shown and hidden. The equipment can also be found via a search function applying the part number or the part name. This will be displayed graphically interactively with the information of the equipment boxes, where is mounted (Fig. 6). 


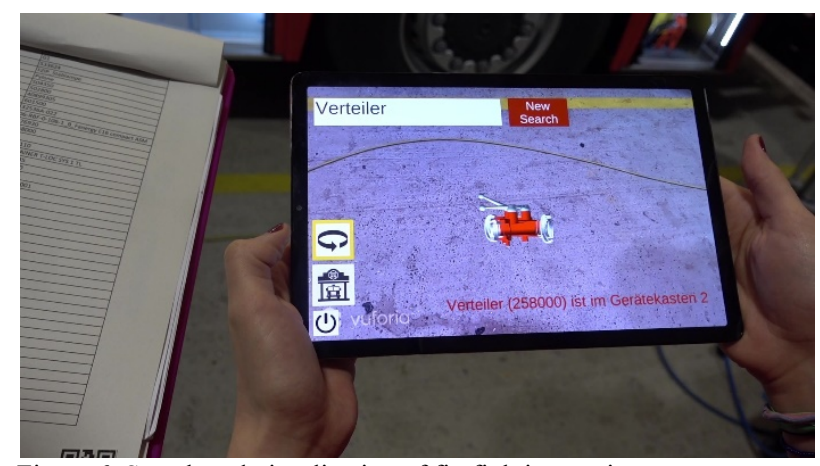

Figure 6. Search and visualization of firefighting equipment

With those implemented AR functions, the employees can visualize the virtual 3D models of the equipment in interactive way in order to get a better view about the equipment box before starting the assembly process. Moreover, the AR application can also be used to check the assembly process regarding the correctness and the completeness of the fitted equipment.

\section{IMPLEMENTATION OF THE AR-BASED ASSEMBLY ASSISTANT APPLICATION}

The AR application was implemented as android app that can be installed and used on mobile devices such as tablets and smartphones as AR device.

For the implementation of the AR application different software applications were used. With the "Unity 3D" the UI including the AR functions were designed and implemented. "Vuforia SDK" was used to generate different graphic targets for the real equipment boxes based on the 3D CAD data models (Fig. 7).

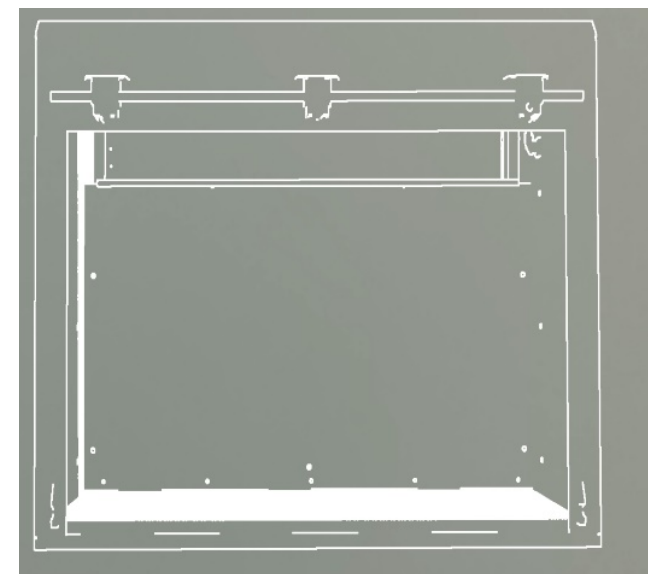

Figure 7. Creation of a target model for the firefighting equipment box

The graphic targets enable the recognition and tracking of the real objects by the camera of the AR devices in the $3 \mathrm{D}$ space. The preparation of the $3 \mathrm{D}$ graphic data for the to be used and rendered in an AR environment was done with the graphic software application "Maxon Cinema4D".

\section{EVALUATION OF THE IMPLEMENTED AR- BASED ASSEMBLY ASSISTANT APPLICATION}

The evaluation of the implemented AR application was done by means of evaluation workshops with assembly employees of Rosenbauer. The evaluation feedback of the involved users can be summed up as follows:

Performance of the AR application: the application offers a stable target recognition of the firefighting equipment boxes. However, the tracking of the equipment boxes can be lost when the user moves very close to the real equipment box. This issue should be tackled in future research works by the use of high-performance tracking sensors and cameras.

Usability of the AR application: The AR application offers a good UX with very interactive, simple and context-oriented functions.

Added value through the use of the AR application: the virtual 3D visualization of the firefighting equipment enables the employees to get an accurate view on the equipment boxes before starting the assembly process. The AR application helps the employees to check the assembly process regarding the correctness and the completeness of the mounted firefighting equipment. So that the error rate can be decreased and the quality issues can be reduced in the future.

\section{HAND TRACKING INTEGRATION IN THE TECHNOLOGY}

We have developed a version for the Realmax Qian devices which is a combined $\mathrm{AR} / \mathrm{VR}$ device using customized Android OS. This device was used with the UltraLeap's Leap Motion hand tracking sensor. The Realmax Qian device observes a $60 \mathrm{~cm}$ diameter hemispherical area with $120 * 150$ degree field of view. The Leap Motion cameras generate almost 200 fps of reflected data making the tracking feel smooth and latency free. The data is analyzed by the Leap Motion software using machine vision algorithms which have not been disclosed by UltraLeap. The device synthesizes 3D hand position by comparing $2 \mathrm{D}$ frames generated by the cameras. The overall average accuracy of the controller is 0.7 millimeters allowing for very precise hand gestures and operations. [20]

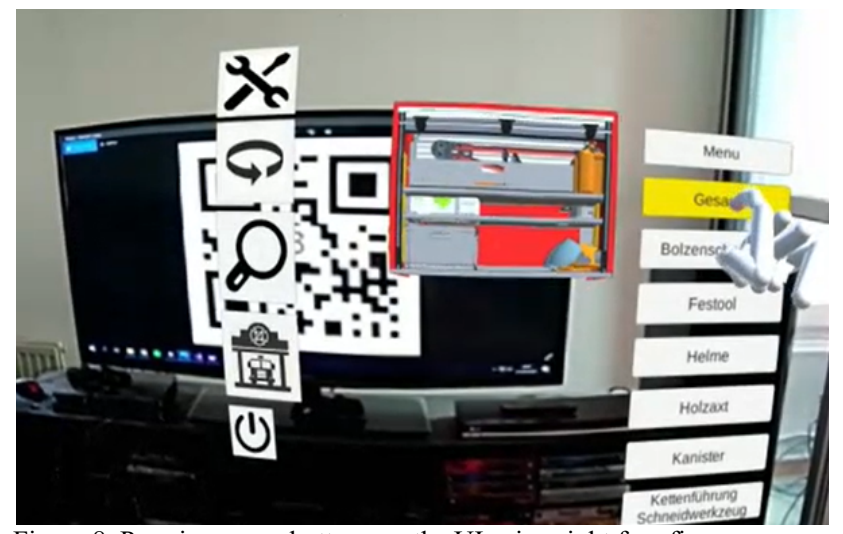

Figure 8. Pressing menu buttons on the UI using right fore finger.

This version enables the virtual visualization of the fire truck boxes with head mounted displays. After identifying a QR code firefighting equipment box is identified and the user is able to select assemblies pointing UI elements with right or left for fingers (Figure 8 and 9). So the use of this version is quite similar compared to the tablet version presented above. 


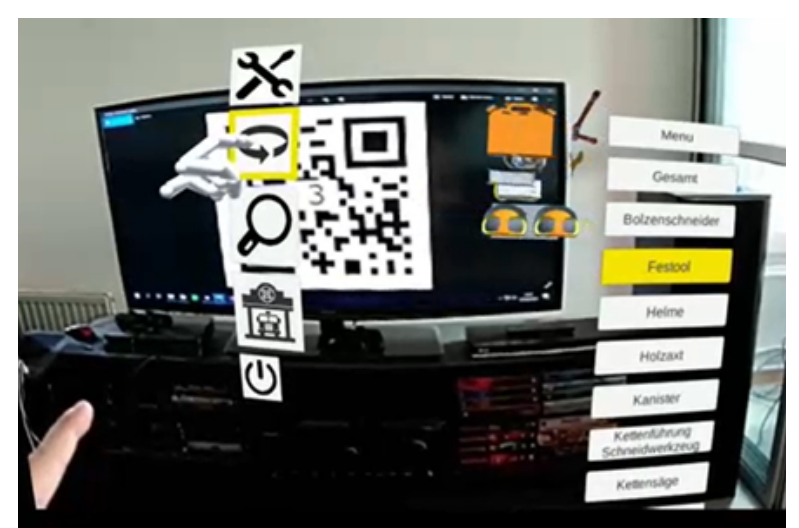

Figure 9. Pressing rotation button on the UI using left fore finger.

The integration of Ultraleap's Motion Leap Controller offers various gestures to track and trace hand and finger movements (touch, press, grab, hold and move). For example zooming could be replaced by using two fore fingers in the same time or pinching with one hand. Realmax Qian device can also be used as VR headsets so this can enlarge the use of this device from office environments to on site environments.

\section{SPEECH RECOGNITION INTEGRATION IN THE TECHNOLOGY}

Another head mounted display we have used in this paper is RealWear HMT-1 device. This device has also Android OS is mainly used based on speech recognition to avoid pressing or tapping any control buttons on the screen. The RealWear HMT-1 device has four digital microphones with active noise cancellation and accurate voice recognition even in $95 \mathrm{dBA}$ of typical industrial noise. In addition, it has internal $91 \mathrm{~dB}$ loudspeaker [21].

The user is again identifying the firefighting equipment box based on QR code. Compared to the original version of the the FiAAR we have included numbers to use English speech recognition which is supported in the HMT-1 device. As visualized in the Figure 10 above equipment can be selected by using numbers in the list.

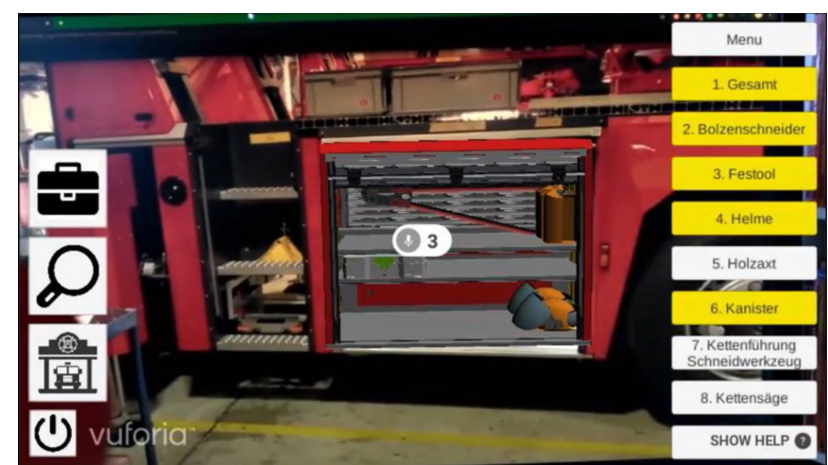

Figure 10. Selecting item number three using speech recognition.

In addition, in this version we are able to communicate with other staff members via video conference. Also different language versions are possible. We have already tested Lingsoft's speech recognition API for Finnish language.

\section{EXPERT EVALUATION.}

The results of the FiAAR application after testing it with internal and external experts quite satisfactory. Rosenbauer users were actually thrilled with the fact that the implementation in AR really worked. It quickly became apparent that this method can really be used to create a virtual interior.

Moreover, communication with the customer was also significantly better due to the AR application. AR visualization also provides value for the employee who carries out the interior work. This is because the AR interior fittings projected into the empty equipment room are a very good guideline for installing the firefighting equipment at the required positions.

External experts evaluated the application from the $\mathrm{UX} / \mathrm{UI}$ perspective and effectiveness on the hands-free interaction utilizing speech recognition and hand recognition functionality. The technology is easy to use and provides realistic images supported with information, instructions, 3D graphics and animation that engages the user. The transparent menus and the actual visualization of the user's virtual hand allows the user to concentrate on the configuration process and learn about the equipment undistractedly.

FiAAR has been developed to educate the user in an indirect pedagogical way while performing the equipment configuration. The integration of designing a solution and learning from it is quite strong in this technology and it seems that this has been achieved quite well.

\section{COMMERCIALIZATION AND MARKET SEGMENT.}

The FiAAR technology is a first initiative to integrate the AR technology in the very niche market of firefighting vehicles. The Rosenbauer firefighting vehicles are designed especially for airport operations. Airport fire trucks meet the highest safety standards and therefore they are considered significant investments for the operations, reputation and safety of the airports. Such demanding clients the expect the best use of their investment need to customization their vehicles to the degree that this can happen.

FiAAR is designed to serve several purposes that will increase the client's satisfaction, standards and expectations. The technology can be commercialized in various ways from both the manufacturer and the clients.

The client can be use FiAAR as a training tool for its firefighting units by training the firefighters on identifying fast and easy the equipment's place on the firefighter truck. This will increase the readiness and decrease the response time in cases of emergency or duty. Such training can be extended to the new firefighters to learn the equipment on the firefighting truck, their identities, usage, locations, assembly instructions etc.

The manufactures can use the technology as tool for the sales engineers towards customizing the firefighting truck to satisfy the client's needs but also help the client configure and design the final product prior proceeding with the purchase investment. Another benefit of the manufacturer from using the FiAAR technology is the extra 
configuration service offered to governmental clients who use strict procurement procedures expecting to assume their investments and protect the tax payers money.

An indirect commercialization route can be achieved through the utilization of the reputational benefits for both the manufacturer and the client. In a world that impression tends to be more important than the essence, staying ahead the competition with aggressive marketing strategies can be very rewarding. Rosenbauer is the first firefighting vehicle manufacturer who integrates AR in its vehicles and its clients are the first to order AR assisted customization and training on the firefighting trucks they purchase.

\section{AREAS OF FURTHER RESEARCH}

The research conducted for the development of the FiAAR technology can be extended on other type of vehicles with related design needs and customer expectations. Rosenbauer provides Municipal Vehicles, Industrial Vehicles, Special Vehicles and Aerials. The concepts developed in FiAAR can also be extended with the integration of VR and Mixed Reality (MR) towards creating more realistic and practical configuration solutions and training scenarios.

Embassies can also be given on specific type of operations aligned with the organizational strategy or the industry demand. Safety is a key issue is all industries with high demand for professional training on any type of vehicles that can serve such purpose.

This can be achieved by following a similar research strategy with the one conducted at TUAS in the maritime sector and the shipping industry in particular for the development of maritime safety VR training applications such as the MarSEVR for command bridge safety training [22], the ShipSEVR for ship engines and engine room safety [23], as well as other safety applications in crane control and firefighting [24]. These maritime VR safety applications were developed with the support of industry partners such as the Meyer Turku shipyard, the Wärtsilä Land and Sea Academy (WLSA) and others.

\section{CONCLUSIONS}

The objective of the presented project is the development of an AR application that enables the virtual visualization the fire truck boxes including the mounted equipment in the $3 \mathrm{D}$ spaces. The implemented $\mathrm{AR}$ application uses a $\mathrm{QR}$ code to identify the firefighting equipment box, which has to be assembled. After detection, the virtual equipment is displayed virtually in the real device box.

The development of the AR application has shown some difficulties in terms of the high implementation effort. The preparation of the 3D graphic of the boxes and the included firefighting equipment and their positioning in the 3D spaces must be done manually within an elaborative and time-consuming process, which needs a high expertise and know-how in the graphic processing tools. For the future, a data transformation process from the CAD to AR platform (e.g. Unity3D) is need that allows an automatic data exchange.
AR devices used in this paper shows the potential current technology can provide for employees working in harsh conditions. Combining speech recognition and hand recognition will free users' hands to be used in other activities. Devices such as HMT-1 can be attached to firemen helmets and it is already a robust solution and widely used in various professions. In the next phase, our plan is to conduct usability evaluation for the developed versions to get better understanding which combination is most preferable in this use case.

One possible future direction is to utilize our previous VR applications and technology presented in this paper. The reuse of assets, animations or even storytelling could generate for us new areas to be explored with our industrial partners. We could for example identify safety challenges in VR and then test how efficiently they can work on site [25]. And at the end again validating in VR whether professionals are able to proceed based on safety instructions.

\section{ACKNOWLEDGEMENT}

The authors would like to thank everyone who have participated in the development of this training VR technology. This work was supported by Business Finland, Turku University of Applied Sciences, University of Applied Sciences Karlsruhe, and Rosenbauer GmbH.

\section{REFERENCES}

[1] Zhou, F.; Duh, H.B.-L.; Billinghurst, M. Trends in augmented reality tracking, interac-tion and display: A review of ten years of ISMAR. In Proceedings of the 7th IEEE/ACM International Symposium on Mixed and Augmented Reality, Cambridge, UK, 15-18 Sep-tember 2008; pp. 193-202.

[2] Felter B. (2019). 5 Use Cases for Edge Computing and Autonomous Vehicles. Vxchange. May 20, 2019. Available at https://www.vxchnge.com/blog/edge-computing-use-casesautonomous-vehicles Accessed on June 2, 2020

[3] Davis C. (2018). Your car windshield could be an AR hologram in 2020. Slashgear. September 10, 2018. Available at: https://www.slashgear.com/porsche-hyundai-wayray-augmentedreality-holographic-windshield-investment-18546322/ Accessed on June 11, 2020

[4] Cortez M. B (2017). Augmented Reality in the Classroom: Transforming Education Through Technology. EdTeck Magazine. 16 October 2017. Available at https://edtechmagazine.com/k12/article/2017/10/how-will-artransform-education-infographic Accessed on June 11, 2020

[5] Yuen S. C-Y., Yaoyuneyong G., and Johnson E. (2011) "Augmented Reality: An Overview and Five Directions for AR in Education," Journal of Educational Technology De-velopment and Exchange (JETDE): Vol. 4 : Iss. 1 , Article 11.

[6] Kerawalla, L., Luckin, R., Selijefot, S., \& Woolard, A. (2006). Making it real: Exploring the potential of augmented reality for teaching primary school science. Virtual Reality, 10(3-4), 163-174

[7] Shelton, B. E., \& Hedley, N. R. (2002). Using augmented reality for teaching earth-sun relationship to undergraduate geography students. The First IEEE International Augmented Reality Toolkit Workshop (pp. 1-8). Darmstadt, Germany: IEEE

[8] Billinghurst, M., and Kato, H. (2002). Collaborative augmented reality. Communica-tions of the ACM, 45(7), 64-70

[9] Quintero, J., Baldiris, S., Rubira, R., Cerón, J., \& Velez, G. (2019). Augmented Reality in Edu-cational Inclusion. A Systematic Review on the Last Decade. Frontiers in psychology, 10, 1835. https://doi.org/10.3389/fpsyg.2019.01835

[10] DigiGapital (2019). For AR/VR 2.0 to live, AR/VR 1.0 must die. January 15,2019 . Available at https://www.digi- 
capital.com/news/2019/01/for-ar-vr-2-0-to-live-ar-vr-1-0-mustdie/. Accessed on June 12, 2020

[11] Draper S. (2018). Cardiologists Use Augmented Reality to Plan and Perform Complex Procedures. Wearable Technologies. November 12, $2010 \quad$ Available at https://www.wearabletechnologies.com/2018/11/cardiologists-use-augmented-reality-toplan-and-perform-complex-procedures/ Accessed on June 10, 2020

[12] Munihan T. (2016). It's a Good Thing the F-35's \$400K Helmet Is Stupid Cool. WIRED. 06 October 2016. Available at https://www.wired.com/2016/06/course-f-35-comes-400000augmented-reality-helmet/ Accessed on June 12, 2020

[13] Nitendosoup (2019) Pokemon Go officially hits 1 billion downloads worldwide. Availa-ble at :https://nintendosoup.com/pokemon-goofficially-hits-1-billion-downloads-worldwide/ Accessed on June 12,2020

[14] Buckle C. (2019) AR vs VR: The Challenges and Opportunities in 2019. Global Web In-dex. 19 November 2018.Available at : https://blog.globalwebindex.com/chart-of-the-week/augmentedvirtual-reality/ Accessed on June 10, 2020

[15] Deloite (2019). 2019 mid-market technology trends report. Deloite. Available at : https://www2.deloitte.com/us/en/pages/deloitteprivate/articles/technology-trends-middle-market-companiessurvey.html Accessed on June 18, 2020

[16] Seal A. (2020). Top 7 Augmented Reality Statistics for 2020. Vxchange. 23 January, 2020. Available at : https://www.vxchnge.com/blog/augmented-reality-statistics Accessed on June 6, 2020.

[17] Hills-Duty R. (2018). Microsoft HoloLens Sales Figures Revealed VRFocus. August 1, 2018. Available at: https:/www.vrfocus.com/2018/05/microsoft-hololens-salesfigures-revealed/ Accessed on June 11, 2020

[18] Marashi A. (2018). Building a Better Data Center With Predictive Analytics. VXchange. August 14, 2018. Available at :https://www.vxchnge.com/blog/better-data-center-withpredictive-analytics. Accessed on June 3, 2020

[19] Angelist (2020). Angelist Community. (N.D). Available at : https://angel.co/ Accessed on June 7, 2020

[20] LeapMotion Datasheet, 2019 , https://www.ultraleap.com/datasheets/Leap_Motion_Controller_D atasheet.pdf

[21] RealWear

HMT-1 specification https://www.realwear.com/knowledge-center/hmt-1/productoverview/specifications/

[22] Markopoulos, E., Lauronen, J., Luimula, M., Lehto, P. and Laukkanen, S. Maritime safety education with VR technology (MarSEVR). In Proceedings of the 9th IEEE Conference on Cognitive Infocommunications, Naples, Italy, 23-25 October 2019; pp. 283-288.

[23] Markopoulos, E., Luimula, M., Porramo, P., Pisirici, T., and Kirjonen. A. Virtual Reali-ty (VR) safety education for ship engine training on maintenance and safety (ShipSEVR). In Proceedings of the 11th International Conference on Applied Human Factors and Ergonom-ics (AHFE), San Diego, USA, 16-20 July 2020.

[24] Markopoulos, E. and Luimula, M. Immersive Safe Oceans Technology: Developing Virtual Onboard Training Episodes for Maritime Safety. Future Internet 2020, 12, 80.

[25] Oliva, D., Somerkoski, B., Tarkkanen, K., Lehto A., and Luimula, M. Virtual reality as a communication tool for fire safety Experiences from the VirPa project. In: Proceeding of the 3rd GamiFIN conference, Levi, Finland, 2019, pp. 241-252. 